\title{
Some Issues on the $p$-Laplace Equation in Cylindrical Domains
}

\author{
M. Chipot ${ }^{a}$ and Y. Xie ${ }^{b}$ \\ Received January 2007
}

\begin{abstract}
We investigate the asymptotic behavior of the solution to equations of the $p$-Laplacian type in cylindrical domains becoming unbounded and address some issues regarding the solution in unbounded domains.
\end{abstract}

DOI: $10.1134 /$ S0081543808020235

\section{INTRODUCTION}

Many problems of mathematical physics are set in cylindrical domains. For instance, these are porous media flows in channels, plate theory, elasticity theory, etc. In this note we will address the problem of the $p$-Laplace equation. To be more precise, suppose that $\Omega_{\ell}$ is a two-dimensional domain (for simplicity) pictured in the figure below, and let $u_{\ell}$ be the unique solution to the nonlinear problem

$$
\begin{cases}-\Delta_{p} u_{\ell}=f\left(x_{2}\right) & \text { in } \Omega_{\ell} \\ u_{\ell}=0 & \text { on } \partial \Omega_{\ell}\end{cases}
$$

where $\Delta_{p}$ is the usual $p$-Laplace operator defined as $\Delta_{p} u:=\operatorname{div}\left(|\nabla u|^{p-2} \nabla u\right), p>1$, and $\partial \Omega_{\ell}$ denotes the boundary of $\Omega_{\ell}$. Note that the $p$-Laplace operator reduces to the usual Laplacian when $p=2$. In this note, we mainly consider the case $p \neq 2$. One refers to [2-7] for results in the linear case.

We notice that the data $f$ of (1.1) depends only on the $x_{2}$-variable. Of course, due to the boundary conditions at the ends of the cylinder, $u_{\ell}$ is not a function independent of $x_{1}$. However, one expects that when $\ell \rightarrow+\infty, u_{\ell}$ is close to a function depending on $x_{2}$ only. To be more precise, let $u_{\infty}$ be the solution to

$$
\left\{\begin{array}{l}
-\Delta_{p} u_{\infty}=f\left(x_{2}\right) \quad \text { in }(-1,1) \\
u_{\infty}(-1)=u_{\infty}(1)=0 .
\end{array}\right.
$$

Then a natural question is whether

$$
u_{\ell} \rightarrow u_{\infty}
$$

as $\ell \rightarrow+\infty$ in any bounded subdomain of $\mathbb{R} \times(-1,1)$.

We will investigate such convergence in Section 2. In the following Section 3, some property of the solution to the $p$-Laplace equation in an unbounded domain will be discussed.

Before we go into details, let us quote some useful inequalities:

\footnotetext{
${ }^{a}$ Institut für Mathematik, Abteilung für Angewandte Mathematik, Universität Zürich, Winterthurerstrasse 190, CH-8057 Zürich, Switzerland.

${ }^{b}$ Department of Mathematics, Ecole Polytechnique Fédérale de Lausanne, 1015 Lausanne, Switzerland.

E-mail addresses: m.m.chipot@math.unizh.ch (M. Chipot), yitian.xie@epfl.ch (Y. Xie).
} 


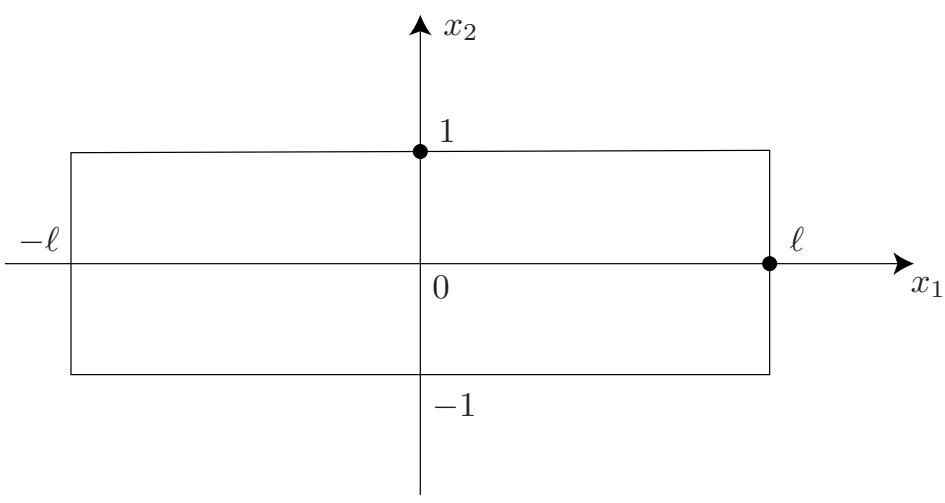

Figure.

Lemma 1.1 (see $[1,8]$ for a proof). For all $p>1, \delta \geq 0$, and $\xi, \eta \in \mathbb{R}^{n}$, it holds that for some constant $c$ depending on $p$

$$
\begin{gathered}
\left.|| \xi\right|^{p-2} \xi-|\eta|^{p-2} \eta|\leq c| \xi-\left.\eta\right|^{1-\delta}(|\xi|+|\eta|)^{p-2+\delta} \\
\left(|\xi|^{p-2} \xi-|\eta|^{p-2} \eta, \xi-\eta\right) \geq c|\xi-\eta|^{2+\delta}(|\xi|+|\eta|)^{p-2-\delta}
\end{gathered}
$$

\section{A CONVERGENCE RESULT}

Let us consider the problem mentioned above in a more general setting. Denote by $X=$ $\left(X_{1}, X_{2}\right)=\left(x_{1}, \ldots, x_{q}, x_{q+1}, \ldots, x_{n}\right)$ any point in $\mathbb{R}^{n}$, and let $\Omega_{\ell}=(-\ell, \ell)^{q} \times \omega$, where $\omega$ is an open bounded domain in $\mathbb{R}^{n-q}$.

Assume that $u_{\ell}$ and $u_{\infty}$ are solutions to

$$
\begin{cases}-\Delta_{p} u_{\ell}=f\left(X_{2}\right) & \text { in } \Omega_{\ell} \\ u_{\ell}=0 & \text { on } \partial \Omega_{\ell}\end{cases}
$$

and

$$
\begin{cases}-\Delta_{p} u_{\infty}=f\left(X_{2}\right) & \text { in } \omega \\ u_{\infty}=0 & \text { on } \partial \omega\end{cases}
$$

respectively. We consider here the weak solutions to (2.1) and (2.2) and assume $u_{\ell} \in W_{0}^{1, p}\left(\Omega_{\ell}\right)$ and $u_{\infty} \in W_{0}^{1, p}(\omega)$, where $W_{0}^{1, p}\left(\Omega_{\ell}\right)$ and $W_{0}^{1, p}(\omega)$ stand for the usual Sobolev spaces. We refer, for instance, to [2] for more information regarding these spaces.

Theorem 2.1. Suppose that $f\left(X_{2}\right) \in L^{p^{\prime}}(\omega)$ is nonnegative (or nonpositive for an analogous statement) and $\Omega$ is any bounded subset in $\mathbb{R}^{q} \times \omega$. Then $u_{\ell}$ is a nondecreasing sequence of nonnegative functions bounded above by $u_{\infty}$, and it holds that

$$
u_{\ell} \rightarrow u_{\infty} \quad \text { in } W^{1, p}(\Omega) .
$$

Proof. We notice that $u_{\ell}$, the solution to (2.1), satisfies

$$
\int_{\Omega_{\ell}}\left|\nabla u_{\ell}\right|^{p-2} \nabla u_{\ell} \nabla v \mathrm{~d} x=\int_{\Omega_{\ell}} f v \mathrm{~d} x \quad \forall v \in W_{0}^{1, p}\left(\Omega_{\ell}\right) .
$$

Take $v=u_{\ell}^{-} \in W_{0}^{1, p}\left(\Omega_{\ell}\right)$, the negative part of $u_{\ell}$, in (2.3). We obtain

$$
\int_{\Omega_{\ell}}\left|\nabla u_{\ell}\right|^{p-2} \nabla u_{\ell} \nabla u_{\ell}^{-} \mathrm{d} x=\int_{\Omega_{\ell}} f u_{\ell}^{-} \mathrm{d} x \geq 0,
$$


i.e.,

$$
\int_{\Omega_{\ell}}\left|\nabla u_{\ell}^{-}\right|^{p} \mathrm{~d} x \leq 0
$$

Hence we derive that $u_{\ell}$ is nonnegative. Following the same arguments, one can show that $u_{\infty}$, the solution to $(2.2)$, is nonnegative.

Letting $\ell<\ell^{\prime}$, one has

$$
\begin{aligned}
\int_{\Omega_{\ell}}\left|\nabla u_{\ell}\right|^{p-2} \nabla u_{\ell} \nabla v \mathrm{~d} x=\int_{\Omega_{\ell}} f v \mathrm{~d} x & \forall v \in W_{0}^{1, p}\left(\Omega_{\ell}\right), \\
\int_{\Omega_{\ell^{\prime}}}\left|\nabla u_{\ell^{\prime}}\right|^{p-2} \nabla u_{\ell^{\prime}} \nabla v \mathrm{~d} x=\int_{\Omega_{\ell^{\prime}}} f v \mathrm{~d} x & \forall v \in W_{0}^{1, p}\left(\Omega_{\ell^{\prime}}\right) .
\end{aligned}
$$

We remark that $u_{\ell^{\prime}}$ is nonnegative in $\Omega_{\ell^{\prime}}$. So

$$
\left(u_{\ell}-u_{\ell^{\prime}}\right)^{+} \in W_{0}^{1, p}\left(\Omega_{\ell}\right) \quad\left(\left(u_{\ell}-u_{\ell^{\prime}}\right)^{+} \text {is the positive part of } u_{\ell}-u_{\ell^{\prime}}\right) .
$$

Therefore, one derives from (2.4) and (2.5) that

$$
\int_{\Omega_{\ell}}\left|\nabla u_{\ell}\right|^{p-2} \nabla u_{\ell} \nabla\left(u_{\ell}-u_{\ell^{\prime}}\right)^{+} \mathrm{d} x=\int_{\Omega_{\ell}}\left|\nabla u_{\ell^{\prime}}\right|^{p-2} \nabla u_{\ell^{\prime}} \nabla\left(u_{\ell}-u_{\ell^{\prime}}\right)^{+} \mathrm{d} x .
$$

The above equation (see (1.4)) leads to

$$
\left(u_{\ell}-u_{\ell^{\prime}}\right)^{+}=0
$$

which shows that $\left\{u_{\ell}\right\}$ is a nondecreasing sequence in $\ell$. On the other hand, we have

$$
\left\{\begin{array}{l}
-\Delta_{p} u_{\ell}+\Delta_{p} u_{\infty}=0 \quad \text { in } \Omega_{\ell}, \\
u_{\ell}-u_{\infty}= \begin{cases}-u_{\infty} \leq 0 & \text { on } \partial(-\ell, \ell)^{q} \times \omega \\
0 & \text { on }(-\ell, \ell)^{q} \times \partial \omega\end{cases}
\end{array}\right.
$$

By the weak maximum principle, this implies

$$
0 \leq u_{\ell} \leq u_{\infty} \quad \text { in } \Omega_{\ell}
$$

Consider now a smooth nonnegative function $\rho(x)$ such that

$$
0 \leq \rho \leq 1, \quad \rho \equiv 1 \quad \text { in } \Omega_{\ell_{0}}, \quad \rho \equiv 0 \quad \text { outside } \Omega_{\ell_{0}+1}, \quad|\nabla \rho| \text { is bounded. }
$$

Taking $u_{\ell} \rho^{p}$ in (2.1), we obtain

$$
\int_{\Omega_{\ell}}\left|\nabla u_{\ell}\right|^{p-2} \nabla u_{\ell} \nabla\left\{u_{\ell} \rho^{p}\right\} \mathrm{d} x=\int_{\Omega_{\ell}} f u_{\ell} \rho^{p} \mathrm{~d} x .
$$

Therefore, for some constant $c$ we have

$$
\begin{aligned}
\int_{\Omega_{\ell_{0}+1}}\left|\nabla u_{\ell}\right|^{p} \rho^{p} \mathrm{~d} x & =-p \int_{\Omega_{\ell_{0}+1}}\left|\nabla u_{\ell}\right|^{p-2} \nabla u_{\ell} \nabla \rho u_{\ell} \rho^{p-1} \mathrm{~d} x+\int_{\Omega_{\ell_{0}+1}} f u_{\ell} \rho^{p} \mathrm{~d} x \\
& \leq c \int_{\Omega_{\ell_{0}+1}}\left|\nabla u_{\ell}\right|^{p-1} u_{\ell} \rho^{p-1} \mathrm{~d} x+\int_{\Omega_{\ell_{0}+1}} f u_{\ell} \rho^{p} \mathrm{~d} x .
\end{aligned}
$$


Applying the Young inequality on the right-hand side, we come to

$$
\int_{\Omega_{\ell_{0}+1}}\left|\nabla u_{\ell}\right|^{p} \rho^{p} \mathrm{~d} x \leq \frac{1}{2} \int_{\Omega_{\ell_{0}+1}}\left|\nabla u_{\ell}\right|^{p} \rho^{p} \mathrm{~d} x+c \int_{\Omega_{\ell_{0}+1}} u_{\ell}^{p} \mathrm{~d} x+\int_{\Omega_{\ell_{0}+1}} f u_{\ell} \rho^{p} \mathrm{~d} x
$$

for some constant $c$. Since $(2.7)$ holds, we derive from (2.9) that

$$
\int_{\Omega_{\ell_{0}}}\left|\nabla u_{\ell}\right|^{p} \mathrm{~d} x \leq 2 c \int_{\Omega_{\ell_{0}+1}} u_{\ell}^{p} \mathrm{~d} x+2 \iint_{\Omega_{\ell_{0}+1}} f u_{\ell} \mathrm{d} x \leq 2 c \int_{\Omega_{\ell_{0}+1}} u_{\infty}^{p} \mathrm{~d} x+2 \int_{\Omega_{\ell_{0}+1}} f u_{\infty} \mathrm{d} x \leq C ;
$$

i.e., $\left\{u_{\ell}\right\}$ is uniformly bounded in $\Omega_{\ell_{0}}$. Up to a subsequence of $u_{\ell}$, labeled still by $\ell$, there exists a $u_{0} \in W^{1, p}\left(\Omega_{\ell_{0}}\right)$ vanishing on $\left(-\ell_{0}, \ell_{0}\right) \times \partial \omega$ such that

$$
u_{\ell} \rightarrow u_{0} \quad \text { in } L^{p}\left(\Omega_{\ell_{0}}\right), \quad u_{\ell} \rightarrow u_{0} \quad \text { in } W^{1, p}\left(\Omega_{\ell_{0}}\right)
$$

We remark here that due to the monotonicity of $\left\{u_{\ell}\right\}$, the whole sequence converges to $u_{0}$ in $L^{p}$.

Fixing a positive constant $h$, for any integer $1 \leq i \leq q$ we consider $u_{\ell}\left(X_{1}+h e_{i}, X_{2}\right)$ and $u_{\ell+h}\left(X_{1}, X_{2}\right)$ in the domain $(-\ell, \ell)^{i-1} \times(-\ell-h, \ell-h) \times(-\ell, \ell)^{q-i} \times \omega$. We have

$$
\begin{gathered}
-\Delta_{p} u_{\ell}\left(X_{1}+h e_{i}, X_{2}\right)+\Delta_{p} u_{\ell+h}\left(X_{1}, X_{2}\right)=f\left(X_{2}\right)-f\left(X_{2}\right)=0 \\
\text { in }(-\ell, \ell)^{i-1} \times(-\ell-h, \ell-h) \times(-\ell, \ell)^{q-i} \times \omega,
\end{gathered}
$$

together with the boundary condition

$$
\begin{aligned}
u_{\ell}\left(X_{1}+h e_{i}, X_{2}\right) & -u_{\ell+h}\left(X_{1}, X_{2}\right) \\
& = \begin{cases}0 & \text { on }(-\ell, \ell)^{i-1} \times(-\ell-h, \ell-h) \times(-\ell, \ell)^{q-i} \times \partial \omega, \\
0 & \text { on }(-\ell, \ell)^{i-1} \times\{-\ell-h\} \times(-\ell, \ell)^{q-i} \times \omega, \\
-u_{\ell+h}\left(X_{1}, X_{2}\right) \leq 0 & \text { on }(-\ell, \ell)^{i-1} \times\{\ell-h\} \times(-\ell, \ell)^{q-i} \times \omega .\end{cases}
\end{aligned}
$$

By the maximum principle we have

$$
u_{\ell}\left(X_{1}+h e_{i}, X_{2}\right) \leq u_{\ell+h}\left(X_{1}, X_{2}\right) \quad \text { in } \quad(-\ell, \ell)^{i-1} \times(-\ell-h, \ell-h) \times(-\ell, \ell)^{q-i} \times \omega .
$$

Passing to the limit in $\Omega_{\ell_{0}}$ leads to

$$
u_{0}\left(X_{1}+h e_{i}, X_{2}\right) \leq u_{0}\left(X_{1}, X_{2}\right)
$$

By changing $h$ to $-h$ and noticing that this holds for all $i$, one has

$$
u_{0}\left(X_{1}, X_{2}\right)=u_{0}\left(X_{2}\right)
$$

Now we denote by $\rho$ a smooth nonnegative function such that (this is slightly different from (2.8))

$$
0 \leq \rho \leq 1, \quad \rho \equiv 1 \quad \text { in } \Omega_{\ell_{0}-1}, \quad \rho \equiv 0 \quad \text { outside } \Omega_{\ell_{0}}, \quad|\nabla \rho| \text { is bounded }
$$

Taking $v=\left(u_{\ell}-u_{0}\right) \rho \in W_{0}^{1, p}\left(\Omega_{\ell_{0}}\right)$ as a test function in $(2.1)$, we obtain

$$
\int_{\Omega_{\ell_{0}}}\left|\nabla u_{\ell}\right|^{p-2} \nabla u_{\ell} \nabla\left\{\left(u_{\ell}-u_{0}\right) \rho\right\} \mathrm{d} x=\int_{\Omega_{\ell_{0}}} f\left(u_{\ell}-u_{0}\right) \rho \mathrm{d} x,
$$


i.e.,

$$
\int_{\Omega_{\ell_{0}}}\left|\nabla u_{\ell}\right|^{p-2} \nabla u_{\ell} \nabla\left(u_{\ell}-u_{0}\right) \rho \mathrm{d} x=-\int_{\Omega_{\ell_{0}}}\left|\nabla u_{\ell}\right|^{p-2} \nabla u_{\ell} \nabla \rho\left(u_{\ell}-u_{0}\right) \mathrm{d} x+\int_{\Omega_{\ell_{0}}} f\left(u_{\ell}-u_{0}\right) \rho \mathrm{d} x .
$$

Since $u_{\ell} \rightarrow u_{0}$ in $L^{p}\left(\Omega_{\ell_{0}}\right)$, it follows that

$$
\int_{\Omega_{\ell_{0}}} f\left(u_{\ell}-u_{0}\right) \rho \mathrm{d} x \rightarrow 0
$$

and

$$
\begin{aligned}
& \left.\left.\left|\int_{\Omega_{\ell_{0}}}\right| \nabla u_{\ell}\right|^{p-2} \nabla u_{\ell} \nabla \rho\left(u_{\ell}-u_{0}\right) \mathrm{d} x\left|\leq c \int_{\Omega_{\ell_{0}}}\right| \nabla u_{\ell}\right|^{p-2}\left|\nabla u_{\ell}\right| \cdot\left|u_{\ell}-u_{0}\right| \mathrm{d} x \\
& \quad \leq c\left\{\int_{\Omega_{\ell_{0}}}\left|\nabla u_{\ell}\right|^{p} \mathrm{~d} x\right\}^{1 / p^{\prime}}\left\{\int_{\Omega_{\ell_{0}}}\left|u_{\ell}-u_{0}\right|^{p} \mathrm{~d} x\right\}^{1 / p} \leq C\left\{\int_{\Omega_{\ell_{0}}}\left|u_{\ell}-u_{0}\right|^{p} \mathrm{~d} x\right\}^{1 / p} \rightarrow 0 .
\end{aligned}
$$

Then we can derive from (2.10)

$$
\int_{\Omega_{\ell_{0}}}\left|\nabla u_{\ell}\right|^{p-2} \nabla u_{\ell} \nabla\left(u_{\ell}-u_{0}\right) \rho \mathrm{d} x \rightarrow 0 .
$$

Therefore, one has

$$
\begin{aligned}
\int_{\Omega_{\ell_{0}}}\left\{\left|\nabla u_{\ell}\right|^{p-2} \nabla u_{\ell}-\left|\nabla u_{0}\right|^{p-2} \nabla u_{0}\right\} \nabla\left(u_{\ell}-u_{0}\right) \rho \mathrm{d} x & \\
& =\int_{\Omega_{\ell_{0}}}\left|\nabla u_{\ell}\right|^{p-2} \nabla u_{\ell} \nabla\left(u_{\ell}-u_{0}\right) \rho \mathrm{d} x-\int_{\Omega_{\ell_{0}}}\left|\nabla u_{0}\right|^{p-2} \nabla u_{0} \nabla\left(u_{\ell}-u_{0}\right) \rho \mathrm{d} x \rightarrow 0 .
\end{aligned}
$$

(Recall that $u_{\ell} \rightarrow u_{0}$ in $W^{1, p}\left(\Omega_{\ell_{0}}\right)$.) We find that when $p \geq 2$ (see (1.4)),

$$
\int_{\Omega_{\ell_{0}}}\left|\nabla\left(u_{\ell}-u_{0}\right)\right|^{p} \rho \mathrm{d} x \leq c \int_{\Omega_{\ell_{0}}}\left\{\left|\nabla u_{\ell}\right|^{p-2} \nabla u_{\ell}-\left|\nabla u_{0}\right|^{p-2} \nabla u_{0}\right\} \nabla\left(u_{\ell}-u_{0}\right) \rho \mathrm{d} x \rightarrow 0 .
$$

When $1<p<2$, it holds that

$$
\begin{aligned}
& \int_{\Omega_{\ell_{0}}}\left|\nabla\left(u_{\ell}-u_{0}\right)\right|^{p} \rho \mathrm{d} x \\
& \leq\left\{\int_{\Omega_{\ell_{0}}}\left\{\left|\nabla u_{\ell}\right|+\left|\nabla u_{0}\right|\right\}^{p-2}\left|\nabla\left(u_{\ell}-u_{0}\right)\right|^{2} \rho \mathrm{d} x\right\}^{p / 2}\left\{\int_{\Omega_{\ell_{0}}}\left\{\left|\nabla u_{\ell}\right|+\left|\nabla u_{0}\right|\right\}^{p} \rho \mathrm{d} x\right\}^{(2-p) / 2} \\
& \leq c\left\{\int_{\Omega_{\ell_{0}}}\left\{\left|\nabla u_{\ell}\right|^{p-2} \nabla u_{\ell}-\left|\nabla u_{0}\right|^{p-2} \nabla u_{0}\right\} \nabla\left(u_{\ell}-u_{0}\right) \rho \mathrm{d} x\right\}^{p / 2}\left\{\int_{\Omega_{\ell_{0}}}\left\{\left|\nabla u_{\ell}\right|+\left|\nabla u_{0}\right|\right\}^{p} \rho \mathrm{d} x\right\}^{(2-p) / 2} \\
& \rightarrow 0 .
\end{aligned}
$$


From above, we derive that for $1<p<+\infty$

$$
\int_{\Omega_{\ell_{0}-1}}\left|\nabla\left(u_{\ell}-u_{0}\right)\right|^{p} \mathrm{~d} x \leq \int_{\Omega_{\ell_{0}}}\left|\nabla\left(u_{\ell}-u_{0}\right)\right|^{p} \rho \mathrm{d} x \rightarrow 0
$$

which shows that

$$
u_{\ell} \rightarrow u_{0} \quad \text { in } W^{1, p}\left(\Omega_{\ell_{0}-1}\right)
$$

Therefore, $u_{0}$ satisfies

$$
-\Delta_{p} u_{0}=f
$$

We finally conclude that $u_{0}=u_{\infty}$ since $u_{0}$ is independent of $X_{1}$. This completes the proof of the theorem.

Remark 2.2. Some other convergence results including some rate of convergence are available. We refer the reader to [9] for details.

\section{A LIOUVILLE TYPE THEOREM}

As we have seen at the end of the proof of Theorem 2.1, a key point in the convergence issue is the uniqueness of the solution to

$$
\begin{cases}-\Delta_{p} u_{0}=f\left(X_{2}\right) & \text { in } \mathbb{R}^{q} \times \omega \\ u_{0}=0 & \text { on } \mathbb{R}^{q} \times \partial \omega\end{cases}
$$

when, eventually, some other assumptions on $u_{0}$, like boundedness, are imposed. This has a flavor of the Liouville theorem. In this direction let us prove

Theorem 3.1. Let $u \in L^{\infty}\left(\mathbb{R}^{q} ; L^{p}(\omega)\right)$ be a weak solution to

$$
\begin{cases}-\Delta_{p} u=0 & \text { in } \mathbb{R}^{q} \times \omega, \\ u=0 & \text { on } \mathbb{R}^{q} \times \partial \omega .\end{cases}
$$

Then $u \equiv 0$; i.e., problem (3.2) does not admit any nontrivial solution.

Before turning to the proof of the theorem, let us make our assumptions precise. $L^{\infty}\left(\mathbb{R}^{q} ; L^{p}(\omega)\right)$ denotes the space of functions from $\mathbb{R}^{q}$ with values in $L^{p}(\omega)$ that are essentially bounded. By a weak solution to $(3.2)$ we mean a function $u$ such that, for any domain $(-\ell, \ell)^{q} \times \omega$,

$$
\begin{gathered}
u \in W^{1, p}\left((-\ell, \ell)^{q} \times \omega\right) \cap L^{\infty}\left(\mathbb{R}^{q} ; L^{p}(\omega)\right), \quad u=0 \quad \text { on }(-\ell, \ell)^{q} \times \partial \omega, \\
\int_{(-\ell, \ell)^{q} \times \omega}|\nabla u|^{p-2} \nabla u \nabla v \mathrm{~d} x=0 \quad \forall v \in W_{0}^{1, p}\left((-\ell, \ell)^{q} \times \omega\right) .
\end{gathered}
$$

Let us now prove the above theorem.

Proof of Theorem 3.1. Let $\rho$ be a nonnegative smooth function such that

$$
0 \leq \rho \leq 1, \quad \rho \equiv 1 \quad \text { in }\left(-\frac{1}{2}, \frac{1}{2}\right), \quad \rho \equiv 0 \quad \text { outside }(-1,1), \quad\left|\rho^{\prime}\right| \text { is bounded }
$$

and set $\rho_{\ell}(x)=\rho\left(\frac{x}{\ell}\right), \Pi:=\prod_{i=1}^{q} \rho_{\ell}\left(x_{i}\right)$. We have, if $\Pi^{p}$ denotes the $p$ th power of $\Pi$,

$$
u \Pi^{p} \in W_{0}^{1, p}\left((-\ell, \ell)^{q} \times \omega\right) .
$$


Plugging the above function into (3.3) leads to

$$
\int_{(-\ell, \ell)^{q} \times \omega}|\nabla u|^{p-2} \nabla u \nabla\left(u \Pi^{p}\right) \mathrm{d} x=0 .
$$

Therefore, we derive that

$$
\begin{aligned}
\int_{(-\ell, \ell)^{q} \times \omega}|\nabla u|^{p-2} \nabla u \nabla u \Pi^{p} \mathrm{~d} x & =-\int_{(-\ell, \ell)^{q} \times \omega}|\nabla u|^{p-2} \nabla u \nabla \Pi^{p} u \mathrm{~d} x \\
& =-p \int_{(-\ell, \ell)^{q} \times \omega}|\nabla u|^{p-2} \nabla u \nabla \Pi u \Pi^{p-1} \mathrm{~d} x \\
& \leq \frac{c}{\ell} \int_{(-\ell, \ell)^{q} \times \omega}|\nabla u|^{p-1}|u| \Pi^{p-1} \mathrm{~d} x .
\end{aligned}
$$

Applying the Hölder inequality to the right-hand side of the above inequality, we obtain

$$
\int_{(-\ell, \ell)^{q} \times \omega}|\nabla u|^{p} \Pi^{p} \mathrm{~d} x \leq \frac{c}{\ell}\left\{\int_{(-\ell, \ell)^{q} \times \omega}|\nabla u|^{p} \Pi^{p} \mathrm{~d} x\right\}^{(p-1) / p}\left\{\int_{(-\ell, \ell)^{q} \times \omega}|u|^{p} \mathrm{~d} x\right\}^{1 / p},
$$

which is equivalent to

$$
\int_{(-\ell, \ell)^{q} \times \omega}|\nabla u|^{p} \Pi^{p} \mathrm{~d} x \leq \frac{c}{\ell^{p}} \int_{(-\ell, \ell)^{q} \times \omega}|u|^{p} \mathrm{~d} x
$$

We remark that $u$ vanishes on $\mathbb{R}^{q} \times \partial \omega$, i.e., for a.e. $X_{1}, u\left(X_{1}, \cdot\right) \in W_{0}^{1, p}(\omega)$. Exploiting Poincaré's inequality in the $X_{2}$-direction (see [3]) yields

$$
\int_{(-\ell, \ell)^{q} \times \omega}|\nabla u|^{p} \Pi^{p} \mathrm{~d} x \leq \frac{c}{\ell^{p}} \int_{(-\ell, \ell)^{q} \times \omega}\left|\nabla_{X_{2}} u\right|^{p} \mathrm{~d} x
$$

$\left(\nabla_{X_{2}}=\left(\partial x_{q+1}, \ldots, \partial x_{n}\right)\right)$. Due to the choice that we made for $\Pi$, we obtain

$$
\int_{\left(-\frac{\ell}{2}, \frac{\ell}{2}\right)^{q} \times \omega}|\nabla u|^{p} \mathrm{~d} x \leq \frac{c}{\ell^{p}} \int_{(-\ell, \ell)^{q} \times \omega}|\nabla u|^{p} \mathrm{~d} x .
$$

After iterating $k-1$ times this inequality, it comes that

$$
\int_{\left(-\frac{\ell}{2}, \frac{\ell}{2}\right)^{q} \times \omega}|\nabla u|^{p} \mathrm{~d} x \leq \frac{c}{\ell^{p(k-1)}} \int_{\left(-2^{k-1} \ell, 2^{k-1} \ell\right)^{q} \times \omega}|\nabla u|^{p} \mathrm{~d} x .
$$

Hence we derive from (3.4) that

$$
\int_{\left(-\frac{\ell}{2}, \frac{\ell}{2}\right)^{q} \times \omega}|\nabla u|^{p} \mathrm{~d} x \leq \frac{c}{\ell^{p k}} \int_{\left(-2^{k} \ell, 2^{k} \ell\right)^{q} \times \omega}|u|^{p} \mathrm{~d} x .
$$


Since $u \in L^{\infty}\left(\mathbb{R}^{q} ; L^{p}(\omega)\right)$, we obtain

$$
\int_{\left(-\frac{\ell}{2}, \frac{\ell}{2}\right)^{q} \times \omega}|\nabla u|^{p} \mathrm{~d} x \leq \frac{c}{\ell^{p k}}\left(2^{k+1} \ell\right)^{q} \operatorname{ess} \sup \left|u\left(X_{1}, \cdot\right)\right|_{L^{p}(\omega)}^{p}
$$

(ess sup denotes the essential supremum in $X_{1}$ ). Choosing $k$ so large that $p k>q$ and letting $\ell \rightarrow \infty$ lead to

$$
u=0 .
$$

This completes the proof of the theorem.

Remark 3.2. One should notice that one cannot remove entirely the assumption

$$
u \in L^{\infty}\left(\mathbb{R}^{q} ; L^{p}(\omega)\right)
$$

Indeed, suppose that

$$
p=2, \quad q=1, \quad \omega=(-\pi, \pi) .
$$

Then the function

$$
u=e^{x_{1}} \sin x_{2}
$$

is a nontrivial weak solution to

$$
\begin{cases}-\Delta u=0 & \text { in } \mathbb{R} \times \omega \\ u=0 & \text { on } \mathbb{R} \times \partial \omega\end{cases}
$$

\section{ACKNOWLEDGMENTS}

The authors have been supported by the Swiss National Science Foundation under the contracts \#20-103300/1 and \#20-111543/1.

\section{REFERENCES}

1. J. W. Barrett and W. B. Liu, "Finite Element Approximation of the Parabolic p-Laplacian," SIAM J. Numer. Anal. 31 (2), 413-428 (1994).

2. M. Chipot, Elements of Nonlinear Analysis (Birkhäuser, Basel, 2000).

3. M. Chipot, $\ell$ Goes to Plus Infinity (Birkhäuser, Basel, 2002).

4. M. Chipot and A. Rougirel, "On the Asymptotic Behaviour of the Solution of Elliptic Problems in Cylindrical Domains Becoming Unbounded," Commun. Contemp. Math. 4 (1), 15-44 (2002).

5. M. Chipot and A. Rougirel, "On the Asymptotic Behaviour of the Solution of Parabolic Problems in Cylindrical Domains of Large Size in Some Directions," Discrete Contin. Dyn. Syst. B 1 (3), 319-338 (2001).

6. M. Chipot and A. Rougirel, "Remarks on the Asymptotic Behaviour of the Solution to Parabolic Problems in Domains Becoming Unbounded," Nonlinear Anal., Theory Methods Appl. 47 (1), 3-11 (2001).

7. M. Chipot and Y. Xie, "On the Asymptotic Behaviour of the $p$-Laplace Equation in Cylinders Becoming Unbounded," in Nonlinear Partial Differential Equations and Their Applications: Proc. Int. Conf., Shanghai, China, 2003, Ed. by N. Kenmochi, M. Ôtani, and S. Zheng (Gakkotosho, Tokyo, 2004), pp. 16-27.

8. W. Liu and N. Yan, "Quasi-norm Local Error Estimators for p-Laplacian," SIAM J. Numer. Anal. 39 (1), 100-127 (2001).

9. Y. Xie, "On Asymptotic Problems in Cylinders and Other Mathematical Issues," Thes. (Univ. Zürich, May 2006).

This article was submitted by the authors in English 